ARTICLE

\title{
Quantized thermoelectric Hall effect induces giant power factor in a topological semimetal
}

Fei Han (1) 1,9凶 , Nina Andrejevic ${ }^{2,9}$, Thanh Nguyen ${ }^{1,9}$, Vladyslav Kozii (iD) ${ }^{3,9}$, Quynh T. Nguyen,3, Tom Hogan ${ }^{4}$, Zhiwei Ding (1) 2, Ricardo Pablo-Pedro', Shreya Parjan ${ }^{5}$, Brian Skinner (10 ${ }^{3}$, Ahmet Alatas ${ }^{6}$, Ercan Alp ${ }^{6}$, Songxue Chi ${ }^{7}{ }^{7}$, Jaime Fernandez-Baca (10 ${ }^{7}$, Shengxi Huang ${ }^{(0)}{ }^{8}$, Liang $\mathrm{Fu}^{3 凶}$ \& Mingda Li ${ }^{1 凶}$

Thermoelectrics are promising by directly generating electricity from waste heat. However, (sub-)room-temperature thermoelectrics have been a long-standing challenge due to vanishing electronic entropy at low temperatures. Topological materials offer a new avenue for energy harvesting applications. Recent theories predicted that topological semimetals at the quantum limit can lead to a large, non-saturating thermopower and a quantized thermoelectric Hall conductivity approaching a universal value. Here, we experimentally demonstrate the non-saturating thermopower and quantized thermoelectric Hall effect in the topological Weyl semimetal (WSM) tantalum phosphide (TaP). An ultrahigh longitudinal thermopower $S_{x x} \sim 1.1 \times 10^{3} \mu \mathrm{VK}^{-1}$ and giant power factor $\sim 525 \mu \mathrm{W} \mathrm{cm}^{-1} \mathrm{~K}^{-2}$ are observed at $\sim 40 \mathrm{~K}$, which is largely attributed to the quantized thermoelectric Hall effect. Our work highlights the unique quantized thermoelectric Hall effect realized in a WSM toward low-temperature energy harvesting applications.

\footnotetext{
${ }^{1}$ Department of Nuclear Science and Engineering, Massachusetts Institute of Technology, Cambridge, MA 02139, USA. ${ }^{2}$ Department of Materials Science and Engineering, Massachusetts Institute of Technology, Cambridge, MA 02139, USA. ${ }^{3}$ Department of Physics, Massachusetts Institute of Technology, Cambridge, MA 02139, USA. ${ }^{4}$ Quantum Design, Inc., San Diego, CA 92121, USA. ${ }^{5}$ Department of Physics, Wellesley College, Wellesley, MA 02481, USA. ${ }^{6}$ Advanced Photon Source, Argonne National Laboratory, Lemont, IL 60439, USA. ${ }^{7}$ Neutron Scattering Division, Oak Ridge National Laboratory, Oak Ridge, TN 37831, USA. ${ }^{8}$ Department of Electrical Engineering, The Pennsylvania State University, State College, PA 16802, USA. ${ }^{9}$ These authors contributed

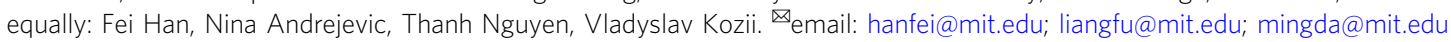


O ver two-thirds of global energy production is rejected as waste heat. Thermoelectrics are attractive by directly converting waste heat into electricity without moving parts. The efficiency of thermoelectric energy conversion is an increasing function of a dimensionless quantity $z T=\sigma S^{2} T / \kappa$, where $\sigma, S$, and $\kappa$ denote the electrical conductivity, thermopower, and total thermal conductivity, respectively ${ }^{1}$. Conventional thermoelectrics largely focus on tuning the thermal and electrical conductivities. Many efforts, such as lowering dimensionality ${ }^{2}$, microstructuring 3,4 , and nanostructuring 5,6 , share the same principle: by increasing the scattering of major heat carriers of long mean free-path phonons without affecting the short mean free-path electrons, a level of independent tunability between electrical conductivity $\sigma$ and thermal conductivity $\kappa$ can be achieved, such as the phonon-glass electron-crystal state ${ }^{7}$. However, less attention was paid to improve the thermopower $S$, even though the $S^{2}$ dependence in $z T$ makes such improvement appealing. Moreover, thermopower $S$ is proportional to the entropy per carrier and is therefore suppressed at reduced temperature $^{8}$. For this reason, current thermoelectrics are generally effective only at elevated temperatures and there is a pressing need for thermoelectrics that work efficiently at room temperature and below. Filling this need requires new materials that can exhibit large electronic entropy at low temperatures while maintaining significant electrical conductivity.

One approach to creating large electronic entropy is bandstructure engineering through low carrier density, partially filled carrier pockets $^{9}$; a similar principle has also been applied to semimetals, such as $\mathrm{Bi}^{10}$, graphite ${ }^{11}$, and most recently Weyl semimetals (WSMs), to explore large entropy at low carrier density ${ }^{12-14}$. However, the electrical conductivity is thereby reduced. Magnetic field offers an additional incentive to dramatically increase the entropy, as the linear field dependence of the density of states (DOS) enables unbounded, macroscopic number of states in each Landau level (LL), yet in conventional thermoelectrics, charge carriers will be localized at high $B$-field due to the cyclotron motion, still resulting in low conductivity. Consequently, increasing power factor $\left(\equiv \sigma S^{2}\right)$ creates a significant challenge as it requires optimization of both $\sigma$ and $S$ under conflicting conditions.

The recent development of topological materials ${ }^{15,16}$, including topological WSMs ${ }^{17}$, offers a new pathway to surpass conventional thermoelectrics that relies on the topological protection of electronic states ${ }^{18,19}$. It is particularly worthy to note that the WSM system has a unique $n=0 \mathrm{LL}$, which has a highly unusual, energy-independent DOS $g(n=0)=N_{f} B e / 4 \pi^{2} \hbar^{2} v_{F}$ increasing linearly with $B$, and therefore can create huge electronic entropy. More importantly, the system remains gapless under high field, thanks to the topological nature of Weyl nodes. Consequently, recent theories predicted a non-saturating thermopower ${ }^{20}$ and quantized thermoelectric Hall conductivity at the quantum limit ${ }^{21}$, where electrons and holes contribute additively to high thermoelectric performance without experiencing localization.

In this work, we carry out high-precision thermoelectric measurements using a centimeter-sized crystal WSM TaP (Fig. 1a, b and Supplementary Notes 1 and 2). The Fermi level is fine-tuned through the synthesis procedure to approach the $n=0$ LL near the W2 Weyl node (Fig. 1g). In this system, giant, nonsaturating longitudinal thermopower $S_{x x}$ is observed, which exhibits linear dependence with $B$-field without saturation. In addition, the signature of the quantized thermoelectric Hall conductivity is observed, where the low-temperature, high-field thermoelectric Hall conductivity $\alpha_{x y} \equiv\left[\rho^{-1} S\right]_{x y}$ approaches a universal curve determined by number of fermion flavors, Fermi velocity, and universal constants. Moreover, evidence of
Wiedemann-Franz (WF) law violation further indicates a breakdown of quasiparticle behaviors. Our work leverages the effects of topology to overcome challenges for low-temperature thermoelectric energy harvesting from a power factor perspective.

\section{Results}

Quantum oscillations. We first present the longitudinal magnetoresistance (MR) data, where Giant MR was observed. At $T<25 \mathrm{~K}$, the $M R \equiv\left(\rho_{x x}(B)-\rho_{x x}(0 \mathrm{~T})\right) / \rho_{x x}(0 \mathrm{~T})$ exceeds $10^{5 \%}$ (Fig. 1c). This is a signature of electron-hole compensation, which is further confirmed by the two-band model fitting of conductivity, with $n_{e}=$ $2.39 \times 10^{19} \mathrm{~cm}^{-3}$ and $n_{h}=2.35 \times 10^{19} \mathrm{~cm}^{-3}$ at $T=2.5 \mathrm{~K}$, along with a high mobility of $\sim 1 \times 10^{5} \mathrm{~cm}^{2} \mathrm{~V}^{-1} \mathrm{~s}^{-1}$ (Supplementary Note 3). The background-subtracted $M R$, denoted $\Delta M R$, exhibits Shubnikov-de Haas oscillations, which are plotted against $1 / B$ to determine oscillation frequencies (Fig. 1d). The Fourier transform of $\Delta M R$ shows two small carrier pockets with low frequency $F_{\alpha}=$ $4 \mathrm{~T}$ and $F_{\beta}=18 \mathrm{~T}$ among four pockets (Supplementary Note 4 and Fig. 1e). The LL fan diagram analysis indicates the two small pockets are at $n=2 \mathrm{LL}$ and $n=0 \mathrm{LL}$, respectively (Supplementary Note 5 and Fig. 1f). The intersections of the linear LL index plots $(-0.037$ for $n=0 \mathrm{LL}$ and +0.065 for $n=2)$ lying between $-1 / 8$ to $+1 / 8$ indicates that the two pockets are both topologically nontrivial $^{22,23}$, from which we attribute the $n=2 \mathrm{LL}$ to the electron pocket of the W1 Weyl node, and the $n=0 \mathrm{LL}$ to the hole pocket of the W2 Weyl node (Fig. 1g). Moreover, we see that the W2 and W1 pockets enter the quantum limit at $B \sim 3.8 \mathrm{~T}$ and $16 \mathrm{~T}$, respectively. There is an alternate way to infer LL. The Weyl fermion dispersion of the $n^{\text {th }} \mathrm{LL}$ at is given by $E_{n}=\operatorname{sgn}(n) v_{F} \sqrt{2 e \hbar B|n|}$, whereas the oscillation frequency $F$ satisfies $F=E_{F}^{2} / 2 e \hbar v_{F}^{2}$. When $E_{n} \sim E_{F}$, we have $F \sim B|n|$. This leads to an agreement between $n=2$ $\mathrm{LL}$ and the measured $F_{\beta}=18 \mathrm{~T}$ at $B \sim 9 \mathrm{~T}$. For $F_{\alpha}$, the low frequency $4 \mathrm{~T}$ suggests an extremely small Fermi surface. Since the spacing between $n=1$ and $n=0$ LLs is given by $E_{1}-E_{0}=v_{F} \sqrt{2 e \hbar B}=E_{F} \sqrt{B / F}$, the condition to reach the $n=0$ LL quantum limit for W2 pocket is met at $B>F_{\alpha}=4 \mathrm{~T}$. This value agrees well with the above LL index analysis.

Non-saturating thermopower and giant power factor. Having determined the carrier characteristics, we carried out thermoelectric measurements using a diagonal offset geometry (Fig. 2a), where the electrical and thermal transport along both the longitudinal and transverse directions can be acquired together by flipping the field polarity (Supplementary Note 6, which also contains the phase relations between various thermoelectric quantities). The longitudinal thermopower $S_{x x}$ is shown in Fig. 2b, where $S_{x x}$ increases over 200-fold compared to its zerofield value, and reaches a giant magnitude of $1.07 \times 10^{3} \mu \mathrm{VK}^{-1}$ without sign of saturation at $B=9 \mathrm{~T}$ and $T=40 \mathrm{~K}$. One prominent feature is that $S_{x x}$ develops a double-peak behavior, which may be attributed to the two types of Weyl nodes: the higher carrier mobility and lower carrier density at the W2 node leads to reduced phonon scattering, and thus the high $S_{x x}$ can persist to higher temperatures. Quantitatively, it has been predicted that for the $n=0$ chiral LL of Weyl electrons, $S_{x x}$ obeys a simple formula $^{20}$ :

$$
S_{x x}=\frac{k_{B}^{2}}{h^{2}} \frac{N_{f}}{12} \frac{T B}{v_{F}^{\text {eff }}\left(n_{h}-n_{e}\right)}
$$

where $N_{\mathrm{f}}$ is the degeneracy of the Weyl nodes, $n_{h}-n_{e}$ is the net carrier density, and $v_{F}^{\text {eff }}$ is an effective Fermi velocity. As TaP has two sets of Weyl nodes with different velocities and energies, in this work we introduce $v_{F}^{\text {eff }}$ as an effective parameter capturing an average Fermi velocity of the system. 
a

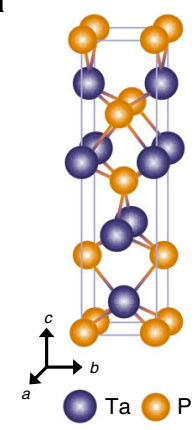

b

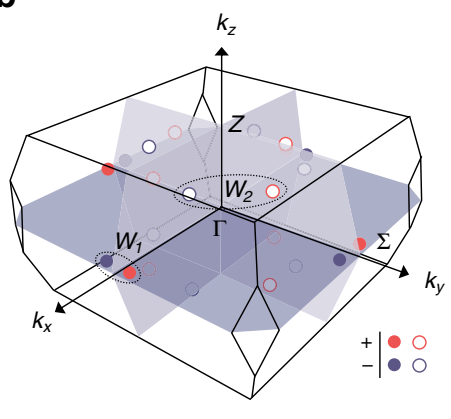

C

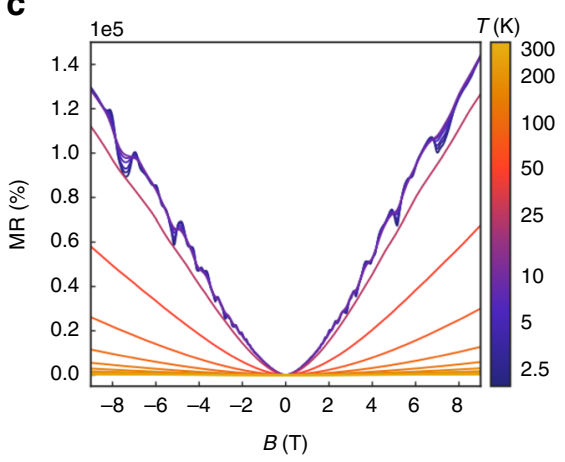

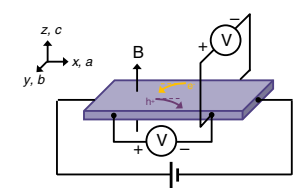

d

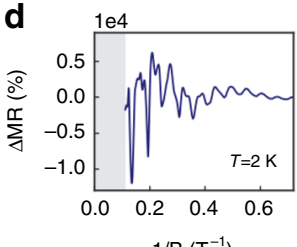

e

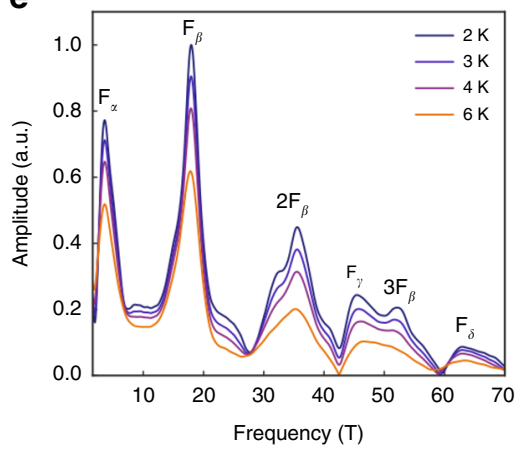

f
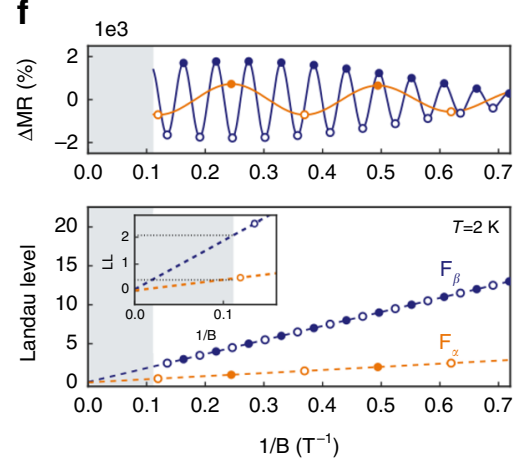

g

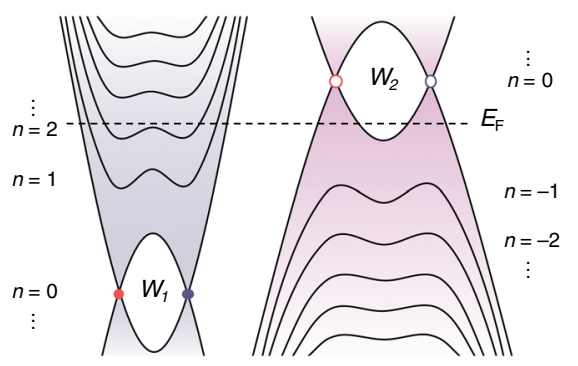

Fig. 1 Quantum oscillation of TaP. a The inversion-symmetry-breaking crystal structure and $\mathbf{b}$ the Brillouin zone of TaP, highlighting the locations of the inequivalent Weyl nodes W1 (filled circles) and W2 (empty circles). The Weyl nodes are paired as source "+" (red) and sink "-" (blue) of Berry curvature, separated in momentum space. c Magnetoresistance (MR) as a function of magnetic field at different temperatures from $2.5 \mathrm{~K}$ to $300 \mathrm{~K}$. A high (>105\%) MR ratio is observed. d The MR measurement configuration (top) and $\triangle M R$ as a function of $1 / B$ (bottom). $\mathrm{e}^{-}$and $\mathrm{h}^{+}$denote electrons and holes, respectively. $\mathbf{e}$ The Fourier transform of $\Delta M R$ showing a low oscillation frequency $F_{\alpha}=4 \mathrm{~T}$. This is a signature that, in addition to the electron pocket from W1 Weyl node contributing to $F_{\beta}=18 \mathrm{~T}$, we are very close to the W2 Weyl node. $\mathbf{f}$ The SdH oscillation and Landau level index plot, from which we obtained an $n=2$ Landau level and another $n=0$ Landau level. $\mathbf{g}$ The schematic bandstructure at finite magnetic fields of our TaP sample.

The linearity of $S_{x x}$ with $T$ and $B$ is shown in Fig. 2c, d, respectively. It is noteworthy that Eq. (1) is in quantitative agreement with our result if we adopt the fitted value of the $v_{F}^{\text {eff }}$ using Eq. (3) and Eq. (S14), described in greater detail in the following section. Such quantitative agreement is valid across all fields and up to $\sim 40 \mathrm{~K}$ and is a measure of the success of the effective model (Fig. 2e). Moreover, a giant longitudinal power factor $\equiv S_{x x}^{2} / \rho_{x x}$ up to $525 \mu \mathrm{W} \mathrm{cm}{ }^{-1} \mathrm{~K}^{-2}$ is achieved due to the large entropy generated by the linearly-dispersive bands at quantizing magnetic fields, while a low $\rho_{x x}$ is maintained due to the protection of the gapless $n=0 \mathrm{LL}$, evading the typical fate of carrier cyclotron motion and localization under fields ${ }^{20,21}$. In fact, this value is an order of magnitude higher than peak values of promising thermoelectrics (e.g., $10 \mu \mathrm{W} \mathrm{cm} \mathrm{cm}^{-1} \mathrm{~K}^{-2}$ for SnSe at $\left.\sim 800 \mathrm{~K}^{24}\right)$ and two orders of magnitude higher than nontopological semimetals ${ }^{10,11}$, which can achieve high thermopower at high magnetic fields with linear-dispersive bands, but cannot simultaneously maintain a low magneto-electrical resistivity.

Quantized thermoelectric Hall effect. Regarding the transverse properties, we see that the transverse thermopower $S_{y x}$ exhibits a plateau with increasing $B$-field, which is known to originate from the constant $k$-space volume as thermopower is a measure of occupational entropy in state space ${ }^{12}$. The thermoelectric Hall conductivity $\alpha_{x y} \equiv\left(S_{x x} \rho_{y x}-S_{y x} \rho_{x x}\right) /\left(\rho_{x x}^{2}+\rho_{y x}^{2}\right)$ is shown in Fig. $3 \mathrm{~b}$, where in the low-temperature range, the flatness with respect to $B$-field starts to emerge. In particular, under the lowtemperature $k_{B} T \ll E_{F}$ and high-field $B \gg E_{F}^{2} / \hbar e v_{F}^{2}$ limit, $\alpha_{x y}$ is predicted to approach the following universal value that is independent of $B$-field, disorder, carrier density, and even carrier type $^{21}$ :

$$
\alpha_{x y, \text { ideal }}=\frac{\pi^{2}}{3} \frac{e k_{B}^{2}}{(2 \pi \hbar)^{2}} \frac{N_{f}}{v_{F}^{\text {eff }}} T
$$

The temperature dependence of $\alpha_{x y}$ is shown in Fig. 3c, where we see that the linearity with $T$ holds up to $T \sim 10 \mathrm{~K}$. As a direct consequence, the $\alpha_{x y} / T$ curves converge to a single curve at high fields (Fig. 3d, e), where an ideal value $\alpha_{x y}$, ideal $/ T=0.4 \mathrm{~A} \mathrm{~K}^{-2} \mathrm{~m}^{-1}$ is determined by evaluating Eq. (2) using $v_{F}^{\text {eff }}=1.4 \times 10^{4} \mathrm{~m} \mathrm{~s}^{-1}$, which is extracted by fitting a more general Eq. (3) at base temperature:

$$
\alpha_{x y}=\frac{e N_{f}}{2 \pi \hbar} \sum_{n=0}^{\infty} \int_{0}^{\infty} \frac{d k_{z}}{\pi}\left[s\left(\frac{\varepsilon_{n}^{0}\left(k_{z}\right)-\mu}{k_{B} T}\right)+s\left(\frac{\varepsilon_{n}^{0}\left(k_{z}\right)+\mu}{k_{B} T}\right)\right]
$$

in which $s$ is the electronic entropy function (Eq. S13). The magnitude $v_{F}^{\text {eff }}$ is comparable to the simple weighted average of projected Fermi velocity $v_{F, z}^{W 1}=0.77 \times 10^{5} \mathrm{~m} \mathrm{~s}^{-1}, v_{F, z}^{W 2}=1.88 \times 10^{5} \mathrm{~m} \mathrm{~s}^{-125}$, which gives $\bar{v}_{F, z}^{\text {eff }}=1.5 \times 10^{5} \mathrm{~m} \mathrm{~s}^{-1}$, where the $z$-direction was chosen to coincide with the magnetic field direction. The fitted chemical potential $\mu$ is consistent with the electrical transport measurements, whereas the $v_{F}^{\text {eff }}$ is lower than the $v_{F}$ at $\mathrm{W} 2^{25}$. This can be understood, as carriers at W1 Weyl nodes at $n=2 \mathrm{LL}$ have yet to reach extreme quantum limit (Figs. $1 \mathrm{~g}$ and $3 \mathrm{f}$, and Supplementary Notes 6 and 7). For temperatures above $10 \mathrm{~K}$, scattering effects are significant and the dissipationless limit assumed in Eq. (3) is no longer valid; thus, for fits at $T \geq 10 \mathrm{~K}$, approximate forms of $\alpha_{x y}$, which include a 
a
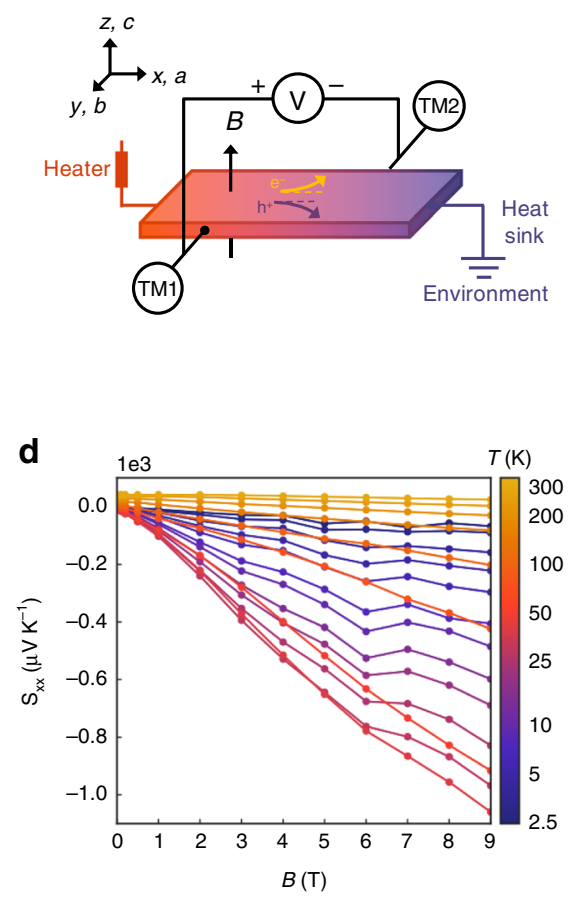

b

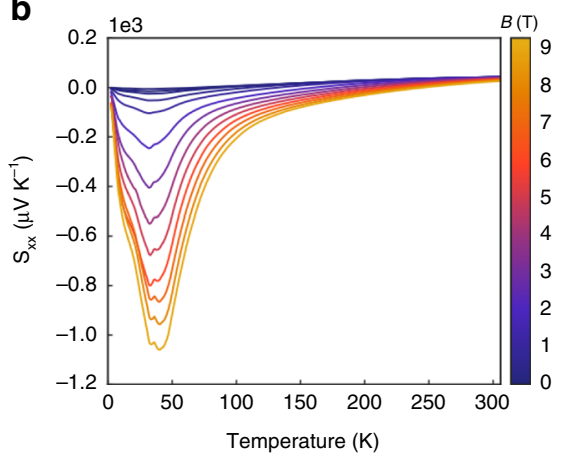

e

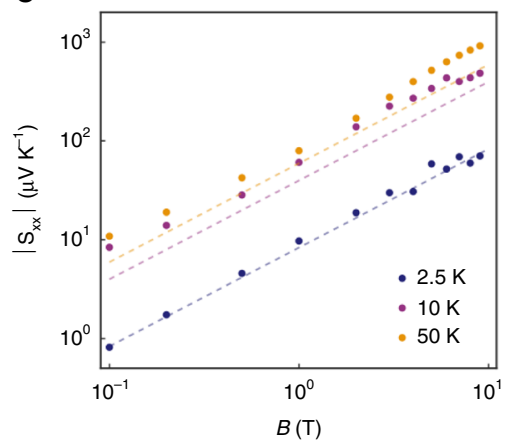

C

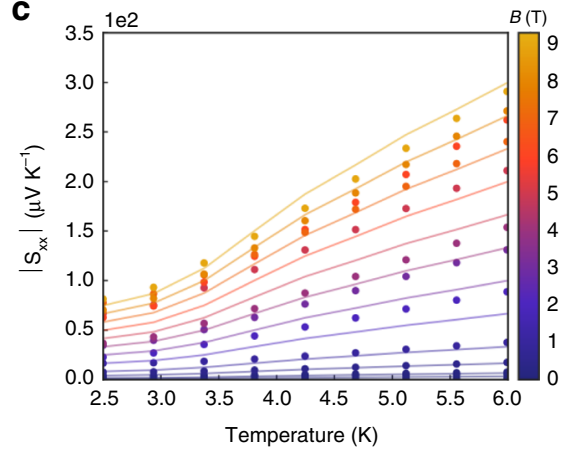

f

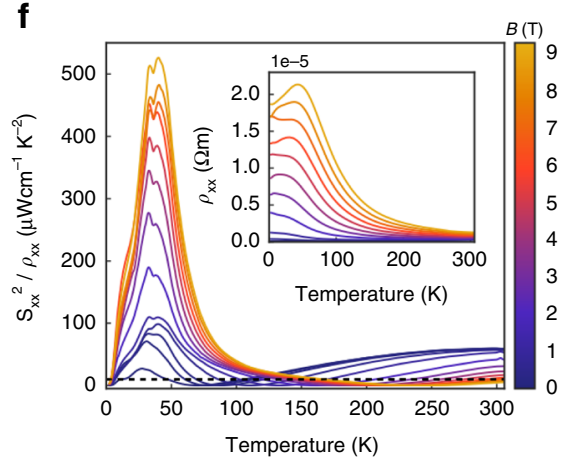

Fig. 2 Non-saturating thermopower at high fields. a The schematics of the diagonal offset thermoelectric measurement geometry. TM1 and TM2 represent thermometer 1 and 2. The temperature difference between the short ends of the sample is represented by the color gradient from red (high) to blue (low). b Longitudinal thermopower $S_{x x}$ as a function of temperature at various fields. The double peaks emerge at $\sim 33 \mathrm{~K}$ and $\sim 40 \mathrm{~K}$. c $S_{x x}$ in the low-temperature range, showing the quasi-linear growth as a function of temperature. $\mathbf{d} S_{x x}$ replotted as a function of $B$, showing unbounded linear growth with field. The onset of the linear behavior indicates entrance into the quantum limit regime. The oscillatory behavior $\sim 20 \mathrm{~K}$ at $B=6 \mathrm{~T}$ is caused by the quantum oscillation effect. e $S_{x x}$ as a function of $B$ at a few representative temperatures. The dashed lines are theoretical values using Eq. (1) by substituting the fitted $v_{F}$ from Eq. (3) (for $T=2.5 \mathrm{~K}$ ) and Eq. (S14) (for $T \geq 10 \mathrm{~K}$ ). $\mathbf{f}$ The power factor as a function of temperature. The black-dashed line is a reference peak value for SnSe.

finite scattering time were used (Eq. (S14) and Eq. (S16)). To corroborate the universal quantization behavior of $\alpha_{x y} / T$, we performed separate thermoelectric measurements up to $B=14 \mathrm{~T}$ at $T=2 \mathrm{~K}, 4 \mathrm{~K}$ and $6 \mathrm{~K}$, where the collapse onto a single curve and a clearer plateau are observed (Supplementary Note 8), in addition to giving $\alpha_{x y}$, ideal $/ T=0.37 \mathrm{~A} \mathrm{~K}^{-2} \mathrm{~m}^{-1}$, in quantitative agreement with the $9 \mathrm{~T}$ data. Finally, to show that the quantized thermoelectric Hall coefficient $\alpha_{x y}$ drives the ultrahigh thermopower and giant power factor, we decompose $S_{x x}$ into its transverse $\left(-\alpha_{x y} \rho_{x y}\right)$ and longitudinal $\left(\alpha_{x x} \rho_{x x}\right)$ components, where we see that the transverse term $\alpha_{x y} \rho_{x y}$ contributes to $\sim 90 \%$ of the longitudinal $S_{x x}$ (Fig. $4 \mathrm{a}$ and Supplementary Note 9 ). The corresponding decomposed contributions to power factor $S_{x x}^{2} / \rho_{x x}$ is shown in Fig. $4 \mathrm{~b}$.

In a nutshell, the quantized $\alpha_{x y}$, large non-saturating $S_{x x}$, and ultrahigh power factor $S_{x x}^{2} / \rho_{x x}$ all originate from the topological Weyl nodes, but with increasingly stringent manifestation conditions: the quantized $\alpha_{x y}$ comes directly from the gapless $n=0$ LL states of Weyl fermions; as $S_{x x}=-\alpha_{x y} \rho_{x y}+\alpha_{x x} \rho_{x x}, \rho_{x y}$ should increase at high fields to obtain non-saturating $S_{x x}$ with the field-independent $\alpha_{x y}$; only when the transverse components $-\alpha_{x y} \rho_{x y}$ dominate the $S_{x x}$ with moderate $\rho_{x x}$ can the power factor $S_{x x}^{2} / \rho_{x x}$ be enhanced; the gapless $n=0 \mathrm{LL}$ states can also contribute to reduce the $\rho_{x x}$.

Breakdown of the Wiedemann-Franz law. The WiedemannFranz law is a robust empirical law stating that the ratio between the electronic thermal conductivity $\kappa^{e}$ and electrical conductivity $\sigma$ are related by a universal Lorenz number:

$$
L_{0} \equiv \frac{\kappa^{e}}{\sigma T}=\frac{\pi^{2}}{3}\left(\frac{k_{B}}{e}\right)^{2}=2.44 \times 10^{-8} \mathrm{~W} \Omega \mathrm{K}^{-2}
$$

Recently, it has been reported that there is strong violation of the WF law in the 2D Dirac fluid of graphene ${ }^{26}$ and WSM WP ${ }_{2}^{27}$ due to collective electron hydrodynamics. Other behaviors of electrons, like quantum criticality ${ }^{28}$ or quasiparticle breakdown ${ }^{29,30}$, can also lead to the WF law violation. It is thus worthwhile to examine the validity of the WF law in the field-induced highentropy state of TaP. To do so, it is crucial to properly separate $\kappa^{e}$ from the lattice thermal conductivity $\kappa^{p h}$. We adopt the following empirical relation by using the field-dependence of $\kappa^{e 31}$ :

$$
\kappa_{x x}(T, B)=\kappa_{x x}^{p h}(T)+\frac{\kappa_{x x}^{e}(T)}{1+\beta_{e}(T) B^{m}}
$$

where $\beta_{e}(T)$ is a measure of zero-field electron mean free path and $m$ is a factor related to the nature of scattering. Figure $5 \mathrm{a}$ demonstrates an example for such a separation procedure (Supplementary Note 11). Using this method, we see that the extracted $\kappa^{p h}$ agrees well with the computed value from ab initio calculations (Fig. 5b and Supplementary Note 12), from which the phonon dispersions are also computed, and agree well with measured dispersion from inelastic scattering (Fig. 5c and Supplementary Note 10). All these agreements indicate the reliability of the separation process. The corresponding $\kappa^{e}$ and the $L_{0}$ is shown in Fig. 5d, e, respectively. At $B=0 \mathrm{~T}$, the agreement with the WF law is good. However, as field increases to $B=9 \mathrm{~T}$, a fourfold violation of WF law is observed (Fig. 5d). This happens 

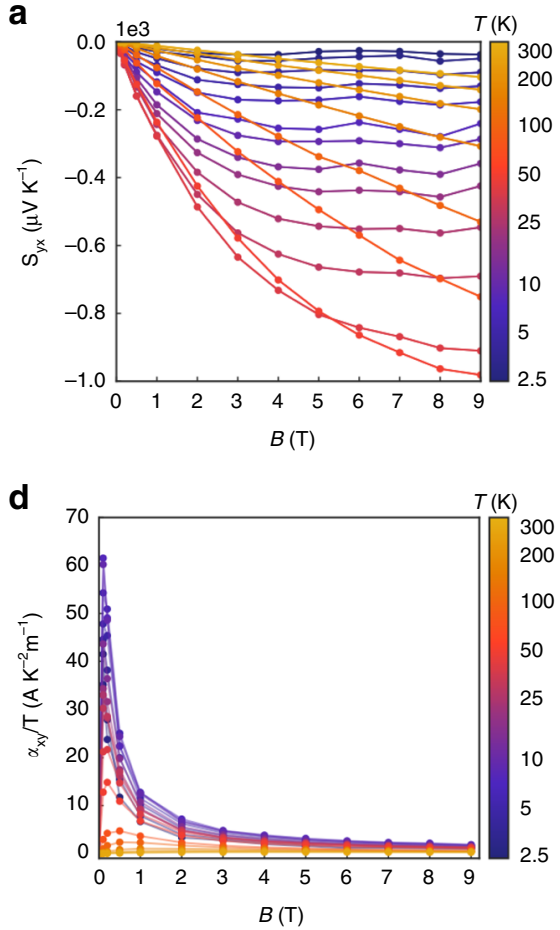

b

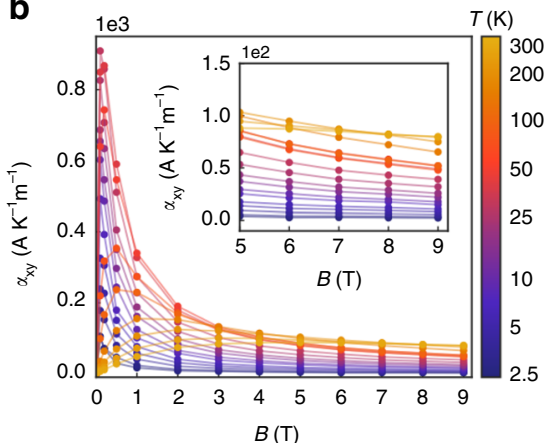

e

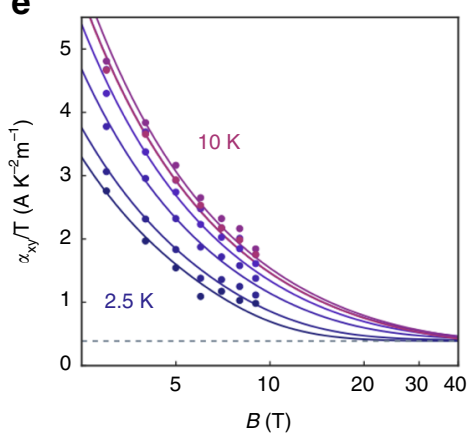

C

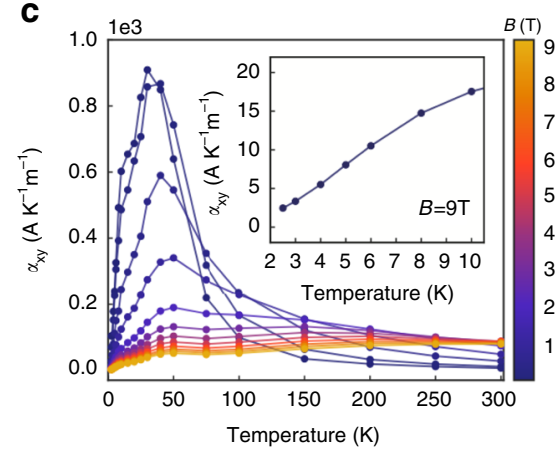

$\mathbf{f}$

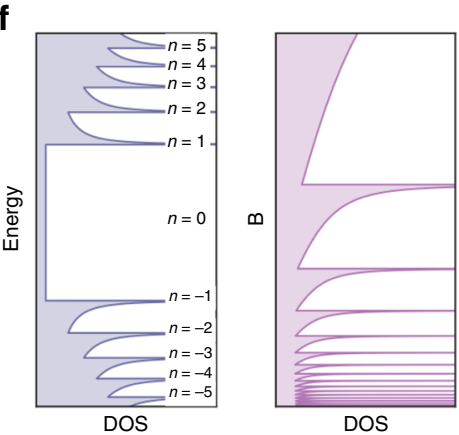

Fig. 3 The quantized thermoelectric Hall effect. a Transverse thermopower $S_{y x}$ as a function of magnetic field at different temperatures. $\mathbf{b}$ Thermoelectric Hall conductivity $\alpha_{x y}$ as a function of magnetic field at different temperatures. The peak value is caused by the finite scattering effect. c Thermoelectric Hall conductivity $\alpha_{x y}$ as a function of temperature at various fields. The inset shows a linear behavior of $\alpha_{x y}$ versus $T$ at low temperatures. $\mathbf{d} \alpha_{x y} / T$ as a function of magnetic field at various temperatures. e An extrapolation of $\mathbf{d}$ showing a convergence to the quantized value at low temperatures. $\mathbf{f}$ The density of states (DOS) of each Landau level (LL), highlighting the unique $n=0 \mathrm{LL}$ in a WSM. At high-enough $B, n=0 \mathrm{LL}$ drives the DOS $\propto B$.
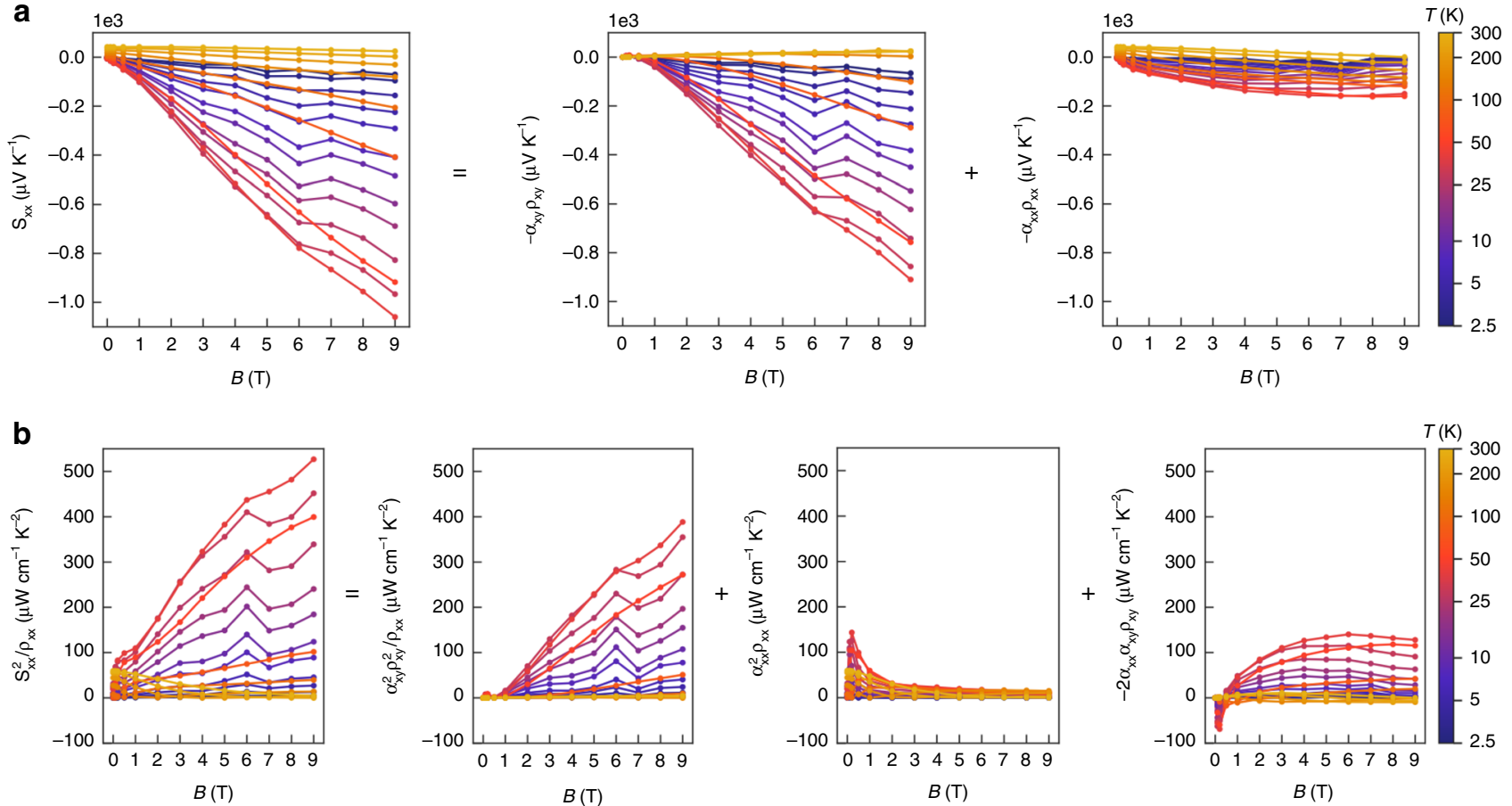

Fig. 4 Dominant contribution from the transverse thermoelectric Hall conductivity to longitudinal thermopower and power factor. a Total $S_{x x}$ (left-hand side, LHS) at various temperatures as a function of magnetic field and its transverse component $-\alpha_{x y} \rho_{x y}$ (first term on the right-hand side, RHS) and longitudinal contribution $+\alpha_{x x} \rho_{x x}$ (second term on the RHS). $\mathbf{b}$ Total $S_{x x}^{2} / \rho_{x x}$ (LHS) at various temperatures as a function of magnetic field and its transverse component $+\alpha_{x y}^{2} \rho_{x y}^{2} / \rho_{x x}$ (first term on RHS), longitudinal contribution $+\alpha_{x x}^{2} \rho_{x x}$ (second term on the RHS), and the cross-term $-2 \alpha_{x y} \alpha_{x x} \rho_{x y}$ (third term on the RHS). The dominant contribution of transverse component can be seen at all temperatures. 
a

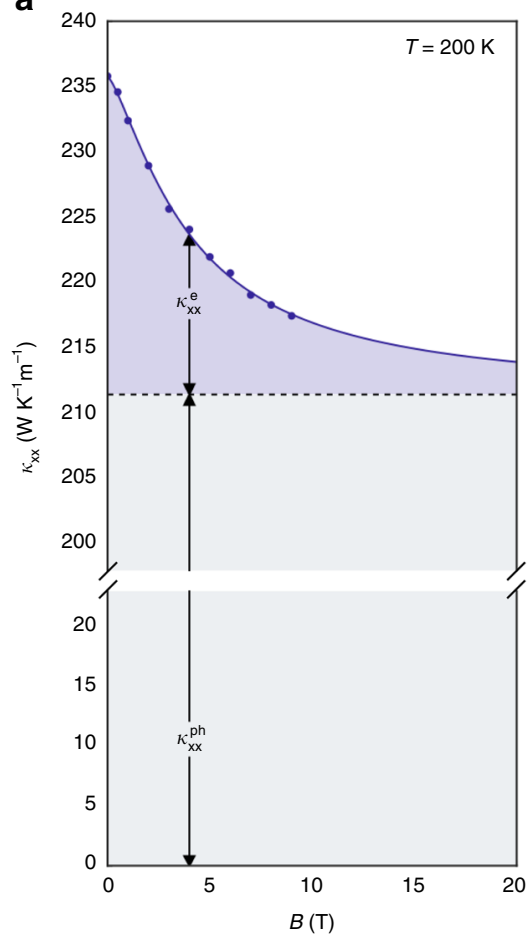

b

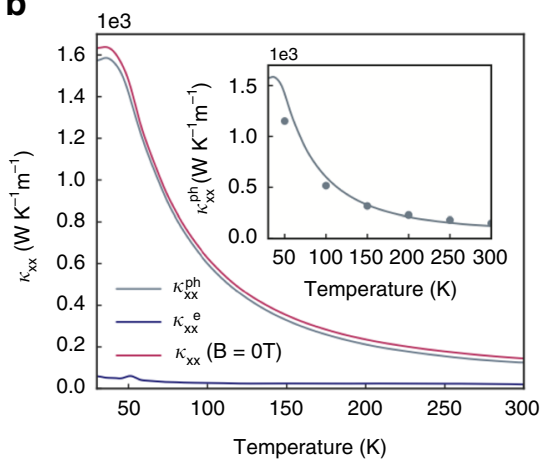

d

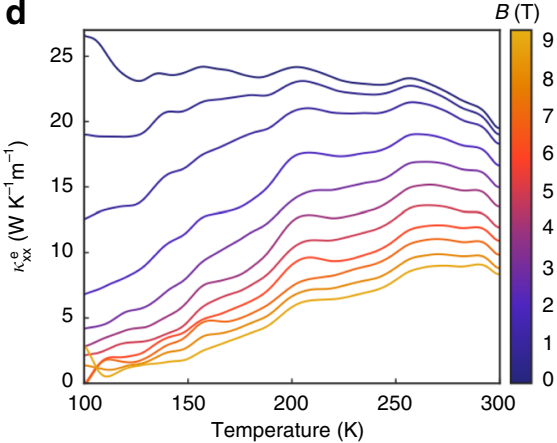

C
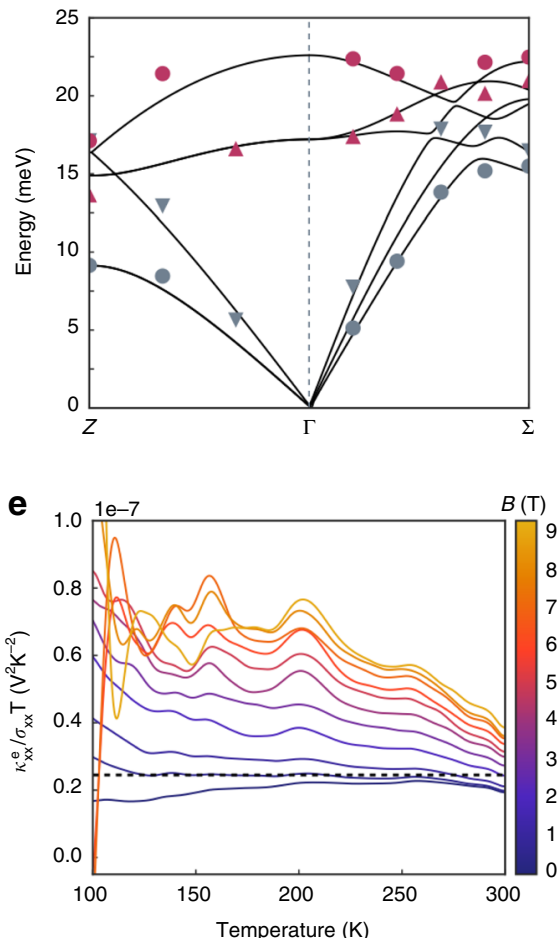

Fig. 5 The Wiedemann-Franz Law. a The schematics of the separation process of electronic thermal conductivity $\kappa_{x x}^{e}$ from the lattice thermal conductivity using $\kappa_{x x}^{p h}$ the field dependence. $\mathbf{b}$ Separation of phonon and electronic contributions to the longitudinal thermal conductivity with inset displaying a computation (scattered points) of the phonon thermal conductivity from first principles. c Experimentally measured values of phonon modes (scattered points) of TaP along high-symmetry line $Z-\Gamma-\Sigma$ taken by inelastic $x$-ray and neutron scattering with accompanying ab initio calculation (solid lines), displaying good agreement between ab initio calculations and experiment. $\mathbf{d}$ The electronic contribution of the thermal conductivity as a function of temperature at various fields. e The Lorenz number as a function of temperature at various fields. The black line indicates the theoretical value of the Wiedemann-Franz law.

across a wide temperature range but not at low temperatures, indicating the link of scattering (Supplementary Note 11). The observed strong violation of the WF law hints at the possibility of field-driven, scattering-enhanced collective behaviors in a large entropic system and is subject to further investigation.

\section{Discussion}

Pathway toward room-temperature topological thermoelectrics. In this work, we report high thermopower and giant thermoelectric power factor in the WSM TaP, induced by the quantized thermoelectric Hall effect originating from topologically protected Weyl nodes at the quantum limit. These features are linked as follows: in a strong magnetic field, $S_{x x} \sim \alpha_{x y} \rho_{y x}$, the quantizing behavior of $\alpha_{x y}$ combined with the continual increase of $\rho_{y x}$ with magnetic field leads to the growth of $S_{x x}$, while the suppression of longitudinal portion $\alpha_{x x} \rho_{x x}$ to $S_{x x}$ further contributes to high power factor $S_{x x}^{2} / \rho_{x x}$. The choice of TaP is due to its simpler Fermi surface compared to other members in the TaAs family ${ }^{25}$. On the other hand, the huge mass difference between $\mathrm{Ta}$ and $\mathrm{P}$ atoms reduces the three-phonon process and results in a high thermal conductivity, making it not directly applicable as a thermoelectric material. Even so, our work sheds light on a systematic pathway to seek promising topological thermoelectrics: To increase $S_{x x}$, large carrier compensation is desired (Eq. (1)). To simultaneously maintain low $\rho_{x x}$, simultaneously high carrier densities $n_{h}$ and $n_{e}$ are required but not sufficient. In a topologically trivial semimetal such as $\mathrm{Bi}$, although high thermopower can be achieved at the quantum limit $\left(S_{x x}(\mathrm{Bi}) \sim 3 \times 10^{3} \mu \mathrm{V} \mathrm{K}^{-1}\right.$ vs $\left.S_{x x}(\mathrm{TaP}) \sim 1 \times 10^{3} \mu \mathrm{V} \mathrm{K}^{-1}\right)$, the electrical resistivity is significantly enhanced at high magnetic field $\left(\rho_{x x}(\mathrm{Bi}) \sim 2 \times 10^{-2} \Omega \mathrm{m} \text { vs } \rho_{x x}(\mathrm{TaP}) \sim 1 \times 10^{-5} \Omega \mathrm{m}\right)^{10}$, indicating the crucial contribution of the gapless $n=0 \mathrm{LL}$ states from the topologically protected Weyl nodes. To tune the working temperature toward room temperature, long relaxation time is favored, along with preservation of the quantum limit, where thermal energy is smaller than the LL level spacing, $k_{B} T \ll v_{F} \sqrt{\hbar e B}{ }^{21}$. Finally, intrinsic magnetism can be used to replace the external $B$-field. Overall, we foresee that magnetic topological WSMs and related topological nodal line semimetals ${ }^{32,33}$ with protected gapless states are promising candidate materials for thermoelectrics when charge carriers are largely compensated and the Fermi level is tuned to the gapless nodes to unlock the quantized thermoelectric Hall effect. To summarize, we demonstrated non-saturating longitudinal thermopower, giant power factor, and a signature of quantized thermoelectric Hall conductivity in a WSM in quantitative agreement with recent theoretical proposals. Furthermore, a field-driven breakdown of the WF law is observed at intermediate temperatures. Given the promising magnitudes of thermopower and power factor, our work sheds light on a few essential requirements that high-performance room-temperature thermoelectrics should meet. These include a way to create giant electronic entropy and reduce carrier density, and a way of evading localization while maintaining high electrical conductivity. Interestingly, the $n=0 \mathrm{LL}$ state with topologically protected Weyl nodes in a WSM satisfies all these requirements. Our work thus demonstrates the possibility of topological materials to lead the breakthrough of thermoelectric materials working below room temperature. 
Note added. When we were finalizing this manuscript, we became aware of a work on Dirac semimetal ${ }^{34}$. The related work and our work mutually strengthened each other on the part of the quantized thermoelectric Hall effect.

\section{Data availability}

The datasets generated during and/or analysed during the current study are available from the corresponding author on reasonable request.

Received: 2 July 2020; Accepted: 3 November 2020;

Published online: 02 December 2020

\section{References}

1. Rowe, D. M. CRC Handbook of Thermoelectrics (CRC Press, 1995).

2. Dresselhaus, M. S. et al. New directions for low-dimensional thermoelectric materials. Adv. Mater. 19, 1043-1053 (2007).

3. Biswas, K. et al. High-performance bulk thermoelectrics with all-scale hierarchical architectures. Nature 489, 414-418 (2012).

4. Il Kim, S. et al. Dense dislocation arrays embedded in grain boundaries for high-performance bulk thermoelectrics. Science 348, 109-114 (2015).

5. Minnich, A. J., Dresselhaus, M. S., Ren, Z. F. \& Chen, G. Bulk nanostructured thermoelectric materials: current research and future prospects. Energy Environ. Sci. 2, 466 (2009).

6. Vineis, C. J., Shakouri, A., Majumdar, A. \& Kanatzidis, M. G. Nanostructured thermoelectrics: big efficiency gains from small features. Adv. Mater. 22, 3970-3980 (2010).

7. Snyder, G. J. \& Toberer, E. S. Complex thermoelectric materials. Nat. Mater. 7, 105 (2008)

8. Heremans, J. P. et al. Enhancement of thermoelectric efficiency in $\mathrm{PbTe}$ by distortion of the electronic density of states. Science 321, 554 (2008).

9. Pei, Y. et al. Convergence of electronic bands for high performance bulk thermoelectrics. Nature 473, 66-69 (2011).

10. Mangez, J. H., Issi, J. P. \& Heremans, J. Transport properties of bismuth in quantizing magnetic fields. Phys. Rev. B 14, 4381-4385 (1976).

11. Zhu, Z., Yang, H., Fauqué, B., Kopelevich, Y. \& Behnia, K. Nernst effect and dimensionality in the quantum limit. Nat. Phys. 6, 26-29 (2009).

12. Caglieris, F. et al. Anomalous Nernst effect and field-induced Lifshitz transition in the Weyl semimetals TaP and TaAs. Phys. Rev. B 98, 201107 (2018).

13. Watzman, S. J. et al. Dirac dispersion generates unusually large Nernst effect in Weyl semimetals. Phys. Rev. B 97, 161404 (2018).

14. Sharma, G., Moore, C., Saha, S. \& Tewari, S. Nernst effect in Dirac and inversion-asymmetric Weyl semimetals. Phys. Rev. B 96, 195119 (2017).

15. Hasan, M. Z. \& Kane, C. L. Colloquium: topological insulators. Rev. Mod. Phys. 82, 3045-3067 (2010).

16. Qi, X.-L. \& Zhang, S.-C. Topological insulators and superconductors. Rev. Mod. Phys. 83, 1057-1110 (2011).

17. Armitage, N. P., Mele, E. J. \& Vishwanath, A. Weyl and Dirac semimetals in three-dimensional solids. Rev. Mod. Phys. 90, 015001 (2018).

18. Heremans, J. P., Cava, R. J. \& Samarth, N. Tetradymites as thermoelectrics and topological insulators. Nat. Rev. Mater. 2, 17049 (2017).

19. Lundgren, R., Laurell, P. \& Fiete, G. A. Thermoelectric properties of Weyl and Dirac semimetals. Phys. Rev. B 90, 165115 (2014).

20. Skinner, B. \& Fu, L. Large, nonsaturating thermopower in a quantizing magnetic field. Sci. Adv. 4, eaat2621 (2018).

21. Kozii, V., Skinner, B. \& Fu, L. Thermoelectric Hall conductivity and figure of merit in Dirac/Weyl materials. Phys. Rev. B 99, 155123 (2019).

22. Murakawa, H. et al. Detection of Berry's phase in a bulk Rashba semiconductor. Science 342, 1490 (2013).

23. Zhao, Y. et al. Anisotropic Fermi surface and quantum limit transport in high mobility three-dimensional Dirac semimetal $\mathrm{Cd}_{3} \mathrm{As}_{2}$. Phys. Rev. X 5, 031037 (2015)

24. Zhao, L. D. et al. Ultralow thermal conductivity and high thermoelectric figure of merit in SnSe crystals. Nature 508, 373-377 (2014).

25. Lee, C.-C. et al. Fermi surface interconnectivity and topology in Weyl fermion semimetals TaAs, TaP, NbAs, and NbP. Phys. Rev. B 92, 235104 (2015).

26. Crossno, J. et al. Observation of the Dirac fluid and the breakdown of the Wiedemann-Franz law in graphene. Science 351, 1058-1061 (2016).

27. Gooth, J. et al. Thermal and electrical signatures of a hydrodynamic electron fluid in tungsten diphosphide. Nat. Commun. 9, 4093 (2018).

28. Tanatar, M. A., Paglione, J., Petrovic, C. \& Taillefer, L. Anisotropic violation of the Wiedemann-Franz law at a quantum critical point. Science 316 , $1320-1322$ (2007).
29. Lee, S. et al. Anomalously low electronic thermal conductivity in metallic vanadium dioxide. Science 355, 371 (2017).

30. Hartnoll, S. A. Theory of universal incoherent metallic transport. Nat. Phys. 11, 54-61 (2014).

31. Ocaña, R. \& Esquinazi, P. Thermal conductivity tensor in $\mathrm{YBa}_{2} \mathrm{Cu}_{3} \mathrm{O}_{7-\mathrm{x}}$ : Effects of a planar magnetic field. Phys. Rev. B 66, 064525 (2002).

32. $\mathrm{Hu}$, J. et al. Evidence of topological nodal-line Fermions in $\mathrm{ZrSiSe}$ and $\mathrm{ZrSiTe}$. Phys. Rev. Lett. 117, 016602 (2016).

33. Fang, C., Weng, H., Dai, X. \& Fang, Z. Topological nodal line semimetals. Chin. Phys. B 25, 117106 (2016).

34. Zhang, W. et al. Observation of a thermoelectric Hall plateau in the extreme quantum limit. Nat. Commun. 11, 1046 (2020).

\section{Acknowledgements}

We thank S.Y. Xu for the helpful discussions. F.H., N.A., T.H., and M.L. thank the support from US DOE BES Award number DE-SC0020148. N.A. acknowledges the support of the National Science Foundation Graduate Research Fellowship Program under Grant number 1122374. T.N. thanks the support from the MIT SMA-2 Fellowship Program and Sow-Hsin Chen Fellowship. V.K., B.S., and L.F. thank support from DOE Office of Basic Energy Sciences, Division of Materials Sciences and Engineering under Award DE-SC0018945. Q.T.N. thanks the support from MIT NSE UROP Program. Z.D. thanks support from DOD Defense Advanced Research Projects Agency (DARPA) Materials for Transduction (MATRIX) program under Grant HR0011-16-2-0041. R.P.-P. thanks the support from FEMSA and ITESM. B.S. is supported by the NSF STC "Center for Integrated Quantum Materials” under Cooperative Agreement number DMR1231319. L.F. is partly supported by the David and Lucile Packard Foundation. This research on neutron scattering used resources at the High Flux Isotope Reactor, a DOE Office of Science User Facility operated by the Oak Ridge National Laboratory. This research used resources of the Advanced Photon Source, a U.S. Department of Energy (DOE) Office of Science User Facility operated for the DOE Office of Science by Argonne National Laboratory under Contract number DE-AC02-06CH11357.

\section{Author contributions}

M.L. and F.H. designed the experiments. V.K., B.S., and L.F. formulated the theory. F.H synthesized the samples and performed the transport measurements, with the help of T.N., Q.T.N., and S.P. N.A., T.N., and F.H. analyzed the data, with contribution from all the authors. T.H. and F.H. carried out additional $14 \mathrm{~T}$ measurements. Z.D. carried out ab initio calculations. N.A., T.N., F.H., R.P.-P., and M.L. along with A.A., E.A., S.C., J.F.-B., and S.H. performed inelastic scattering measurements. M.L., N.A., F.H., and T.N. wrote the paper with input from all authors.

\section{Competing interests}

The authors declare no competing interests.

\section{Additional information}

Supplementary information is available for this paper at https://doi.org/10.1038/s41467020-19850-2.

Correspondence and requests for materials should be addressed to F.H., L.F. or M.L.

Peer review information Nature Communications thanks Katsumi Tanigaki, Jia Zhu, and the other, anonymous, reviewer(s) for their contribution to the peer review of this work.

Reprints and permission information is available at http://www.nature.com/reprints

Publisher's note Springer Nature remains neutral with regard to jurisdictional claims in published maps and institutional affiliations.

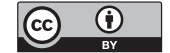

Open Access This article is licensed under a Creative Commons Attribution 4.0 International License, which permits use, sharing, adaptation, distribution and reproduction in any medium or format, as long as you give appropriate credit to the original author(s) and the source, provide a link to the Creative Commons license, and indicate if changes were made. The images or other third party material in this article are included in the article's Creative Commons license, unless indicated otherwise in a credit line to the material. If material is not included in the article's Creative Commons license and your intended use is not permitted by statutory regulation or exceeds the permitted use, you will need to obtain permission directly from the copyright holder. To view a copy of this license, visit http://creativecommons.org/ licenses/by/4.0/.

(C) The Author(s) 2020 